\title{
Effect of constant heat flux at outer cylinder on stability of viscous flow in a narrow-gap annulus with radial temperature gradient
}

\author{
S. Pandey ${ }^{1 *}$, A.K. Singh ${ }^{2}$, R. Prasad ${ }^{3}$ \\ $I^{I^{*}}$ Department of Mathematics, Banaras Hindu University, Varanasi, INDIA \\ ${ }^{2}$ Department of Mathematics, Banaras Hindu University, Varanasi, INDIA \\ ${ }^{3}$ Department of Mathematics, Banaras Hindu University, Varanasi, INDIA \\ "Corresponding Author: e-mail: sadhana.bhu20@gmail.com, Tel+91-9044798212
}

\begin{abstract}
In this paper, the stability of the Couette flow of a viscous incompressible fluid between two concentric rotating cylinders is studied in the presence of a radial temperature gradient, when the outer cylinder is maintained at a constant heat flux. The analytical solution of the eigen-value problem is obtained by using the trigonometric series method, when the gap between the cylinders is narrow. The numerical values of the critical wave number and critical Taylor number are computed from the obtained analytical expressions for the first, second and third approximations. It is found that the difference between the numerical values of the critical Taylor number corresponding to the second and third approximations are very small as compared to the difference between first and second approximations. The critical Taylor numbers obtained by the third approximation agree very well with the earlier results computed numerically by using the shooting method. This clearly indicates that for the better result one should obtain the numerical values by taking more approximations. Also, the amplitude of the radial velocity and the cell-pattern are shown on the graphs for different values of the ratio of the angular velocities.
\end{abstract}

Keywords: Radial temperature gradient, trigonometric series method, constant heat flux, Taylor number.

DOI: http://dx.doi.org/10.4314/ijest.v7i2.1

\section{Introduction}

The stability of flow phenomenon of a viscous incompressible fluid between two concentric rotating cylinders with the inner cylinder rotating or both rotating was first studied by (Taylor, 1923). He used a parameter called Taylor number $T a$ and found that the flow becomes unstable, when the Taylor number exceeds its critical value $T a_{c}$. To determine $T a_{c}$ different methods were given by Taylor (1923), Chandrasekhar (1953), DiPrima (1953), Duty and Reid (1964) and Harris and Reid (1964). In all these papers, it was basically assumed that the two cylinders are at the same temperature and as a result of which radial temperature gradient does not exist. However, in many chemical, electrical and mechanical engineering applications the temperature of both cylinders cannot remain the same. Thus, due to the change in the temperature of both cylinders, there exist a temperature gradient and the stability of the fluid flow is affected by the temperature gradient.

The effects of a radial temperature gradient on the stability of the flow of a viscous fluid was theoretically as well as experimentally studied by Chandrasekhar (1954), Bjorklund and Kays (1959), Hass and Nissan (1961), Yih (1961), Beeker and Kays (1962), Ho et al. (1964), Aoki et al. (1967), Sharman et al. (1973), Walowit et al. (1964), Soundalgekar et al. (1981), Takhar et al. (1985), Singh et al. (1994) and others. Further, the effect of radial temperature gradient on the stability of Dean flow was investigated by Ali et al. (1998) under narrow gap approximation and the effect of radial temperature gradient on the circular Couette flow was analysed by Mutabazi et al. (2001). The Taylor-Dean flow through a curved duct of square cross section, in which walls of the duct except the outer wall rotate around the center of curvature and an azimuthal pressure gradient was imposed, was analyzed by Yamamoto et al. (2004). After that, Soleimani and Sadeghy (2011) investigated numerically the 
stability of the Bingham fluids in Taylor-Dean flow between two concentric cylinders at arbitrary gap spacing. The three dimensional linear stability analysis of the Couette flow between two axial cylinders for shear-thinning fluids with and without yield stress was performed by Alibenyahia et al. (2012).

In all of these studies, it was assumed that the two cylinders are mentioned at the different temperatures. In technological applications, there is another situation in which the outer cylinder is maintained at constant temperature while the inner cylinder can get heated from the heat generated by an external source of energy. Mathematically this situation is studied by imposing a variable or constant heat flux at the inner cylinder while the outer cylinder is maintained at a constant temperature. The stability of flow formation between two concentric rotating cylinders, when there is a constant heat flux at the inner cylinder while the outer one is maintained at a constant temperature was studied by Takhar et al. (1988a), Prasad et al. (2012), Takhar et al. (1988b) for the narrow gap case and wide gap case, respectively. Recently, Mahapatra et al. (2013) studied the effect of radial temperature gradient on the stability of Taylor-Dean flow between two arbitrarily spaced concentric cylinders.

Instead of a constant heat flux at the inner cylinder, we can apply the constant heat flux at the outer cylinder and then find its effect on the stability of flow. This was studied by Eagles et al. (1997) for wide gap case and Takhar et al. (1992) for narrow gap case and solved the eigen-value problem by the shooting method. Stability of pure pressure maintained flow in a curved channel is studied by Pandey and Prasad (2012) and same problem is also discussed by Prasad and Pandey (2012) in the presence of axial magnetic field. A linear stability analysis for the Taylor-Dean flow, between concentric horizontal cylinders with a constant azimuthal pressure gradient, keeping the cylinders at different temperatures, when the inner cylinder is rotating and outer one is stationary was studied by Deka and Paul (2013) for the narrow gap case. Here, our aim is to study the narrow-gap Taylor-stability problem in the case when there is a constant heat flux at the outer cylinder while the inner one is maintained at a constant temperature. We have solved this problem by using the trigonometric series method and the results are compared with those obtained by Takhar et al. (1992) by using a numerical method. Also the amplitude of the radial velocity and the cell-pattern are shown on graphs for different values of the ratio of the angular velocities.

\section{Mathematical Analysis}

Consider the flow of an incompressible viscous fluid between two concentric rotating cylinders of radii $R_{1}$ and $R_{2}\left(R_{1}\right.$, radius of the inner cylinder; $R_{2}$, radius of the outer cylinder) with angular velocities $\Omega_{1}$ and $\Omega_{2}$ respectively. T is the temperature of the fluid and $T_{1}$ is the constant temperature of the inner cylinder respectively. Assuming stationary marginal state, the following differential equations have been obtained to govern the stability of the viscous flow in a narrow-gap annular-space (Takhar et al., 1992):

$$
\begin{aligned}
& \left(D^{2}-a^{2}\right)^{2} u=-a^{2} T_{a}\left[g(x) v+N \cdot(g(x))^{2} \theta\right], \\
& \left(D^{2}-a^{2}\right) v=u \\
& \left(D^{2}-a^{2}\right) \theta=u .
\end{aligned}
$$

with boundary conditions

where,

$$
\begin{aligned}
& u=D u=v=\theta=0 \text { at } x=0, \\
& u=D u=v=D \theta=0 \text { at } x=1 .
\end{aligned}
$$

$$
\begin{aligned}
& d=R_{2}-R_{1}, x=\left(r-R_{1}\right) / d, D=\frac{d}{d x}, \\
& a=\lambda d, \mu=\Omega_{2} / \Omega_{1}, \alpha=\mu-1, g(x)=1+\alpha x, \operatorname{Pr}=v / K, \\
& \theta=2 A K T /\left(\Omega_{1} \operatorname{Pr} q R_{2}\right), R a=\operatorname{Pr} \Omega_{1}^{2} d^{4}\left(q R_{2}\right) \beta /\left(v^{2} K\right), u=\left(v / 2 A d^{2}\right) u, \\
& N=-\left\{\operatorname{Pr}\left(\frac{q R_{2}}{K}\right) \beta \Omega_{2}\right\} /(4 A)=R a / T a, T a=-\left(4 A \Omega_{1} d^{4}\right) / v^{2} .
\end{aligned}
$$

Here, $R a$ and $T a$ are respectively, the Rayleigh number and the Taylor number. 
As suggested by Chandrasekhar (1953, we take a sine series for $v$ in order to satisfy the boundary conditions given by Eq. (4) as follows:

$$
v=\sum_{m=1}^{\infty} A_{m} \sin (m \pi x)
$$

Substituting Eq. (6) in Eq. (2) and then with the help of Eq. (2) and (3), we obtain the value of $\theta$. Using these values of $\theta$ and $v$ in Eq. (1), we have obtained the general solution for $u$ as follows:

$$
\begin{aligned}
& u=-a^{2} T a \sum_{m=1}^{\infty} \frac{A_{m}}{K_{1}^{2}}\left[\left(A_{1}^{(m)}+x A_{2}^{(m)}\right) \cos h(a x)+\left(A_{3}^{(m)}+x A_{4}^{(m)}\right) \sin h(a x)+(1+\alpha x) \sin (m \pi x)+\right. \\
& \left.\frac{4 m \alpha \pi \cos (m \pi x)}{K_{1}}\{1+N(1+\alpha x)\}+N\left\{\left((1+\alpha x)^{2}+\frac{4 \alpha^{2} K_{2}}{K_{1}^{2}}\right) \sin (m \pi x)+\frac{(-1)^{m+1} m \pi K_{1}^{2} x^{2} K_{4}}{48 a^{5} K_{*}}\right\}\right] .
\end{aligned}
$$

where, $A_{1}^{(m)}, A_{2}^{(m)}, A_{3}^{(m)}, A_{4}^{(m)}$ are the constants of integration. In view of the boundary conditions (4) and solving the resulting equations, we have

$$
\begin{aligned}
& A_{1}^{(m)}=-\frac{4 m \pi \alpha(1+2 N)}{K_{1}} \\
& A_{2}^{(m)}=\frac{m \pi}{\Delta}\left[\alpha_{m} \sin K_{0}^{2} a-a \gamma_{m} K_{0}-a \alpha K_{5} \delta_{m}+a^{2}\left(\beta_{m}-\lambda_{m} \mu_{m}\right)\right], \\
& A_{3}^{(m)}=\frac{m \pi}{\Delta}\left[a \alpha_{m}-\gamma_{m} K_{0}-\alpha K_{5} \delta_{m}+a \beta_{m}-a \lambda_{m} \mu_{m}\right] \\
& A_{4}^{(m)}=-\frac{m \pi}{\Delta}\left[K_{6}+\mu_{m} a\left(K_{7}+K_{8}\right)\right]
\end{aligned}
$$

where,

$$
\begin{aligned}
& K_{*}=\cosh (a), K_{0}=\sinh (a), K_{1}=m^{2} \pi^{2}+a^{2}, K_{2}=a^{2}-5 m^{2} \pi^{2}, K_{3}=a^{2}-m^{2} \pi^{2}, \\
& K_{4}=a^{2}\left(6+\alpha^{2} x^{2}+4 \alpha x\right) \sin h(a x)-(\alpha x+3) \cos h(a x), K_{5}=a K_{*}+K_{0}, \\
& K_{6}=\alpha_{m} C_{1}+C_{2}\left(\gamma_{m}+\beta_{m} K_{*}\right)+a^{2} \beta_{m} K_{0}+\frac{4 a^{2} \alpha(-1)^{m+1}(1+2 N(\alpha+1)) K_{0}}{K_{1}} \\
& C_{1}=\left(a-K_{0} K_{*}\right), C_{2}=\left(a K_{*}-K_{0}\right), \alpha_{m}=1+N+\frac{12(-1)^{m+1} N K_{3} \alpha^{2}}{K_{1}^{2}}, \\
& \beta_{m}=\frac{4 \alpha(1+2 N)}{K_{1}}, \Delta=\left(K_{0}^{2} a-a^{2}\right), \\
& \gamma_{m}=(-1)^{m+1}(\alpha+1)(1+(\alpha+1) N)+\frac{12(-1)^{m+1} N K_{3} \alpha^{2}}{K_{1}^{2}}-\beta_{m} K_{*}, \\
& \delta_{m}=-\frac{4(-1)^{m+1}(1+2 N(1+\alpha))}{K_{1}^{2}}-\frac{(-1)^{m+1} N K_{1}^{2}}{48 a^{5}}\{4 a(3+\alpha)-9 \alpha \tan h a\}, \\
& \lambda_{m}=K_{0}^{2} a\left(\alpha^{2}\left(a^{2}+3 a K_{*}+18\right)-6 a^{2}+4 \alpha a\left(a+6 K_{*}\right)\right), \\
& \mu_{m}=\frac{(-1)^{m+1} N K_{1}^{2}}{48 a^{6} K_{*}} .
\end{aligned}
$$


By inserting the mathematical expressions of $u$ and $v$ from Eqs. (7) and (6), respectively, in Eq. (2), we have

$$
\begin{aligned}
& \sum_{\mathrm{n}=1}^{\infty} A_{n} R \sin (n \pi x)=-a^{2} T a \sum_{m=1}^{\infty} \frac{A_{m}}{K_{1}^{2}}\left[\left(A_{1}^{(m)}+x A_{2}^{(m)}\right) \cos h(a x)+\left(A_{3}^{(m)}+x A_{4}^{(m)}\right) \sin h(a x)+R_{1}\right. \\
& \left.+N\left(R_{2}+R_{3}\right)\right] .
\end{aligned}
$$

Multiplying Eq. (12) by $\sin (m \pi x)$ and then integrating over the range $0 \leq x \leq 1$, we obtain a system of linear homogeneous equations for the constants and the requirement that these constants are to all zero leads to the following secular equation:

$$
\begin{aligned}
& \| \frac{n \pi}{R}\left[A_{1}^{(m)} R_{5}+A_{2}^{(m)} R_{6}+A_{3}^{(m)}(-1)^{n+1} K_{0}+A_{4}^{(m)} R_{7}\right]+\frac{(-1)^{m+1} m \pi K_{1}^{2} N}{48 a^{5} K_{*}}+R_{8}+R_{9} \\
& +N \alpha^{2} Y_{m n}+\alpha X_{m n}-\frac{R^{3}}{2 a^{2} T a} \delta_{m n} \|=0 .
\end{aligned}
$$

where,

$$
\begin{aligned}
& R=n^{2} \pi^{2}+a^{2}, \\
& R_{1}=(1+\alpha x) \sin (m \pi x)+\frac{4 m \alpha \pi \cos (m \pi x)}{K_{1}}, \\
& R_{2}=(1+\alpha x)^{2} \sin (m \pi x)+\frac{8 m \pi \alpha \cos (m \pi x)}{K_{1}}(1+\alpha x), \\
& R_{3}=\frac{4 \alpha^{2} K_{2} \sin (m \pi x)}{K_{1}^{2}+\frac{(-1)^{m+1} m \pi K_{1}^{2} x^{2} R_{4}}{48 a^{5} \cos h a},} \\
& R_{4}=a^{2}\left(6+\alpha^{2} x^{2}+4 \alpha x\right) \sin h(a x)-(\alpha x+3) \cos h(a x), \\
& R_{5}=1+(-1)^{n+1} K_{*}, R_{6}=(-1)^{n+1}+\frac{2(-1)^{n} a K_{0}}{R}, \\
& \left.R_{7}=(-1)^{n+1} K_{0}+\frac{2 a}{R}\left((-1)^{n} K_{*}-1\right)\right), \\
& R_{8}=3\left(2 a^{2}+3 \alpha^{2}\right) I_{1}+a^{2} \alpha\left(\alpha I_{2}+4 I_{5}\right)-4 a \alpha\left(\alpha I_{3}+3 I_{4}\right), \\
& R_{9}=\frac{1}{2}\left[1+N\left\{1+\frac{4 \alpha^{2} K_{2}}{K_{1}^{2}} I_{1}\right\}\right] \delta_{m n}, R_{10}=\mathrm{a}^{2}-\mathrm{n}^{2} \pi^{2}, \\
& R_{11}=\mathrm{n}{ }^{4} \pi^{4}-5 \mathrm{a}^{2}\left(\mathrm{a}^{2}-2 \mathrm{n}^{2} \pi^{2}\right), R_{12}=\mathrm{n}^{2} \pi^{2}-3 \mathrm{a}^{2}, R_{13}=\left(\mathrm{n}^{2}-\mathrm{m}^{2}\right), \\
& R_{13}=3\left(\mathrm{n}^{2} \pi^{2}-2 \mathrm{a}^{2}\right) K_{0}+\mathrm{n}^{2} \pi^{2} K_{*}, R_{14}=\left[(-1)^{n+m}-1\right], \\
& R_{15}=\left[1+(1+\alpha)(-1)^{n+m}\right], R_{16}=\left[(-1)^{n+1}+(1+\alpha)(-1)^{m+1}\right], \\
& I_{1}=\frac{\mathrm{n} \pi}{R^{3}}(-1)^{\mathrm{n}}\left[K_{0}\left\{R^{2}+2\left(3 \mathrm{a}^{2}+\mathrm{n}^{2} \pi^{2}\right)\right\}+4 \mathrm{a} K_{*} R\right], \\
& I_{3}=\frac{\mathrm{n} \pi}{R^{4}}(-1)^{\mathrm{n}}\left[K_{0}\left\{R^{4}+12 R^{2} R_{12}+24 R_{11}\right\}+8 R K_{*}\left\{a R^{2}+12 R_{10}\right\}\right], \\
& \left.I_{*} R^{3}+6 a\left\{\left(4 R_{10}+R^{2}\right) K_{0}+\mathrm{R} K_{*}\left(\mathrm{n}^{2} \pi^{2}-2 \mathrm{a}\right)\right\}\right], \\
& \left.R^{2}\right)
\end{aligned}
$$




$$
\begin{aligned}
& I_{4}=\frac{\mathrm{n} \pi}{R^{4}}(-1)^{\mathrm{n}}\left[-K_{*} R\left(R^{2}-2 R_{12}\right)+4 \mathrm{a} R^{2} K_{0}-2 \mathrm{n} \pi R_{12}\right], \\
& I_{5}=\frac{\mathrm{n} \pi}{R}(-1)^{\mathrm{n}}\left[K_{0}+\frac{2 R_{13}}{R^{2}}+\frac{6 \mathrm{a} K_{*}}{R}\left(1+\frac{4 \mathrm{a}^{2}}{R^{2}}\right)\right]-\frac{24 R_{10} \mathrm{n} \pi \mathrm{a}}{R^{4}}, \\
& \delta_{m n}= \begin{cases}1, & \text { if } m \neq n \\
0, & \text { if } m=n\end{cases} \\
& X_{m n}= \begin{cases}\frac{1}{4}+\frac{\mathrm{N}}{2}, & \text { if } m \neq n \\
\frac{2 \mathrm{mn}\left((-1)^{\mathrm{m}+1}-1\right)(1+2 \mathrm{~N})}{R_{13}}\left[\frac{1}{\pi^{2} R_{13}}-\frac{2}{K_{1}}\right], & \text { if } m \neq n\end{cases} \\
& Y_{m n}= \begin{cases}\frac{1}{6}-\frac{1}{4 \mathrm{~m}^{2} \pi^{2}}-\frac{2}{K_{1}}, & \text { if } m \\
\frac{4 \mathrm{mn}(-1)^{\mathrm{m}+1}}{R_{13}}\left[\frac{1}{\pi^{2} R_{13}}-\frac{2}{K_{1}}\right], & \end{cases}
\end{aligned}
$$

On substituting the values of $A_{1}^{(m)}, A_{2}^{(m)}, A_{3}^{(m)}, A_{4}^{(m)}$ and $I_{1}, I_{2}, I_{3}, I_{4}, I_{5}$ from Eqs. (8) - (11), and (14) respectively and taking $N=0$ we have,

$$
\begin{aligned}
& \| \frac{4 \mathrm{~m} n \pi^{2} R_{14} \alpha}{R K_{1}}+\frac{2 \mathrm{am} n \pi^{2} C_{1}}{R^{2} \Delta}\left\{R_{15}+R_{16}+\frac{4 \mathrm{a} \alpha K_{0}}{K_{1} C_{1}}\left[K_{0}+a(-1)^{m+1}\right] R_{14}\right\}+\frac{1}{2} \delta_{m n}+\alpha X_{m n} \\
& -\frac{R^{3}}{2 a^{2} T a} \delta_{m n} \|=0 .
\end{aligned}
$$

For $m=n=1$ we get,

$$
T a=\frac{2}{(\mu+1)} \cdot \frac{\left(\pi^{2}+\mathrm{a}^{2}\right)^{3}}{\mathrm{a}^{2}\left[1-\frac{16 \pi^{2} \mathrm{acosh}^{2}(\mathrm{a} / 2)}{\left(\pi^{2}+\mathrm{a}^{2}\right)^{2}\left(K_{0}+\mathrm{a}\right)}\right]} .
$$

Eqs. (15) and (16) are the result obtained by (Chandrashekhar, 1961).

\section{Result and Discussion}

The numerical value of $T a_{c}$ computed from Eq. (13) corresponding to the first, second and third approximations are listed in Table 1. In this table $T a_{1}, T a_{2}, T a_{3}$ represent the numerical values corresponding to the first, second and third approximations, while $T a_{c}$ is the values obtained by Takhar et al. (1992). This table clearly shows that the values of $T a_{c}$ obtained by the third approximation agree very well with the values obtained numerically by Takhar et al. (1992) using the shooting method. When $\mu$ is positive or negative i.e., the two cylinders are either co-rotating or counter rotating, the value of $T a_{c}$ is observed to decrease with increasing $N$ and hence flow gets destabilized owing to increasing value of $N$, when the two cylinders are either corotating or counter rotating. In the case of co-rotating cylinders, when $\mu$ increases (due to increasing the rotational speed of outer cylinder) $T a_{c}$ decreases. But in the presence of counter rotating cylinders, when the angular speed of outer cylinder increases as 
compared to that of inner cylinders, the flow gets more and more stable, because the value of $T a_{c}$ is observed to increase by increasing $\mu$.

Table 1: Value of critical Taylor and wave numbers

\begin{tabular}{|c|c|c|c|c|c|c|}
\hline$\mu$ & $N$ & $a_{i}$ & $T a_{1}$ & $T a_{2}$ & $T a_{3}$ & $T a_{c}$ \\
\hline \multirow[t]{5}{*}{0.0} & 1.0 & 2.977 & 2066.7246 & 2052.55 & 2052.55 & 2057.5 \\
\hline & 0.75 & 3.005 & 2296.6501 & 2281.02 & 2281.02 & 2282.8 \\
\hline & 0.50 & 3.037 & 2583.1528 & 2560.16 & 2560.16 & 2562.7 \\
\hline & 0.25 & 3.077 & 2949.6938 & 2933.41 & 2933.41 & 2919.6 \\
\hline & 0.00 & 3.127 & 3434.3928 & 3412.97 & 3412.97 & 3390.0 \\
\hline \multirow[t]{5}{*}{0.25} & 1.0 & 2.929 & 1525.9376 & 1526.02 & 1535.63 & 1530.9 \\
\hline & 0.75 & 2.962 & 1718.2650 & 1716.74 & 1724.73 & 1720.5 \\
\hline & 0.50 & 3.003 & 1965.0648 & 1961.94 & 1966.18 & 1962.8 \\
\hline & 0.25 & 3.053 & 2292.6948 & 2288.85 & 2288.85 & 2283.1 \\
\hline & 0.00 & 3.120 & 2747.4587 & 2738.23 & 2738.23 & 2725.3 \\
\hline \multirow[t]{5}{*}{0.50} & 1.0 & 2.887 & 1167.1129 & 1166.86 & 1175.92 & 1175.2 \\
\hline & 0.75 & 2.925 & 1331.6896 & 1332.27 & 1339.94 & 1338.2 \\
\hline & 0.50 & 2.972 & 1549.2590 & 1550.63 & 1555.69 & 1552.8 \\
\hline & 0.25 & 3.035 & 1849.6797 & 1847.75 & 1846.04 & 1847.3 \\
\hline & 0.00 & 3.118 & 2289.5393 & 2284.67 & 2384.67 & 2275.4 \\
\hline \multirow[t]{5}{*}{0.75} & 1.0 & 2.851 & 918.7109 & 909.09 & 909.09 & 926.3 \\
\hline & 0.75 & 2.892 & 1061.1198 & 1061.57 & 1068.26 & 1067.9 \\
\hline & 0.50 & 2.944 & 1254.7781 & 1254.71 & 1260.40 & 1259.5 \\
\hline & 0.25 & 3.016 & 1532.6458 & 1533.74 & 1534.45 & 1532.7 \\
\hline & 0.00 & 3.118 & 1962.4639 & 1960.78 & 1946.66 & 1951.5 \\
\hline \multirow[t]{5}{*}{1.0} & 1.0 & 2.820 & 740.4821 & 714.29 & 769.23 & 746.7 \\
\hline & 0.75 & 2.861 & 864.6610 & 833.33 & 833.33 & 870.5 \\
\hline & 0.50 & 2.918 & 1037.9725 & 1036.91 & 1041.99 & 1042.5 \\
\hline & 0.25 & 2.998 & 1295.8606 & 1295.34 & 1297.19 & 1296.7 \\
\hline & 0.00 & 3.119 & 1717.1574 & 1718.31 & 1714.09 & 1707.8 \\
\hline \multirow[t]{5}{*}{-0.25} & 1.0 & 3.030 & 2923.0413 & 2871.09 & 2871.09 & 2866.4 \\
\hline & 0.75 & 3.050 & 3214.9263 & 3151.59 & 3151.59 & 3148.6 \\
\hline & 0.50 & 3.076 & 3570.8037 & 3491.62 & 3491.62 & 3491.9 \\
\hline & 0.25 & 3.107 & 4013.7922 & 3913.89 & 3913.89 & 3918.2 \\
\hline & 0.00 & 3.145 & 4579.7085 & 4518.75 & 4518.75 & 4461.4 \\
\hline \multirow[t]{5}{*}{-0.50} & 1.0 & 3.080 & 4352.3574 & 4168.40 & 4168.40 & 4151.0 \\
\hline & 0.75 & 3.103 & 4794.1382 & 4564.13 & 4564.13 & 4554.3 \\
\hline & 0.50 & 3.129 & 5334.11621 & 5063.29 & 5065.86 & 5043.2 \\
\hline & 0.25 & 3.161 & 6009.129883 & 5659.31 & 5659.31 & 5647.8 \\
\hline & 0.00 & 3.199 & 6875.7700 & 6406.15 & 6406.15 & 6413.8 \\
\hline \multirow[t]{5}{*}{-0.75} & 1.0 & 3.108 & 6834.3462 & 6218.91 & 6222.78 & 6231.1 \\
\hline & 0.75 & 3.169 & 7835.2312 & 6988.12 & 6993.01 & 6967 \\
\hline & 0.50 & 3.236 & 9185.72461 & 7968.13 & 7980.85 & 7880.5 \\
\hline & 0.25 & 3.312 & 11075.3740 & 9242.14 & 9250.69 & 9032.5 \\
\hline & 0.00 & 3.407 & 13901.3730 & 10882.6 & 10888.5 & 11361.8 \\
\hline \multirow[t]{5}{*}{-1.0} & 1.0 & 2.989 & 10638.8349 & 9115.77 & 9165.90 & 9507.0 \\
\hline & 0.75 & 3.276 & 14869.6768 & 11348.2 & 11429.9 & 11297.3 \\
\hline & 0.50 & 3.567 & 23751.3926 & 13020.8 & 13132.0 & 13380.5 \\
\hline & 0.25 & 3.808 & 50519.9844 & 16460.9 & 16622.3 & 15794.5 \\
\hline & 0.00 & 3.999 & -1754513792.00 & 18331.8 & 18508.2 & 18662.9 \\
\hline
\end{tabular}


Other interesting phenomenon is to know the behavior of the amplitude of the radial velocity and the corresponding cellpattern. So for a set of values of $a_{c}$ and $T a_{c}$, the values of $A_{2}^{(m)} / A_{1}^{(m)}, A_{3}^{(m)} / A_{1}^{(m)}, A_{4}^{(m)} / A_{1}^{(m)}$ are determined from Eq.(4). The eigen-functions thus obtained are normalised so that the amplitude of the radial component of the velocity perturbation is unity. These eigen-functions $u(x)$ and the corresponding cell-patterns for the stream function $\Psi=\mathrm{u}(\mathrm{x}) \cos \left(a_{c} \mathrm{z}\right)$ at the onset of instability for different values of $\mu$ and $N$ are shown in Figs. 1-3.

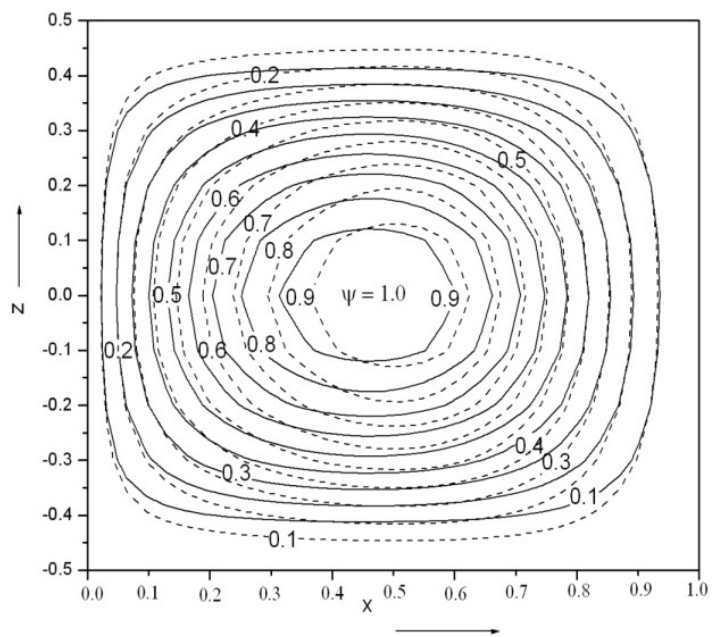

Fig.1. Comparison of the cell patterns at the onset of instability for $N=0.75$ (shown by continuous curve) and $N=0.25$ (shown by broken curve) at constant $\mu=0.75$.

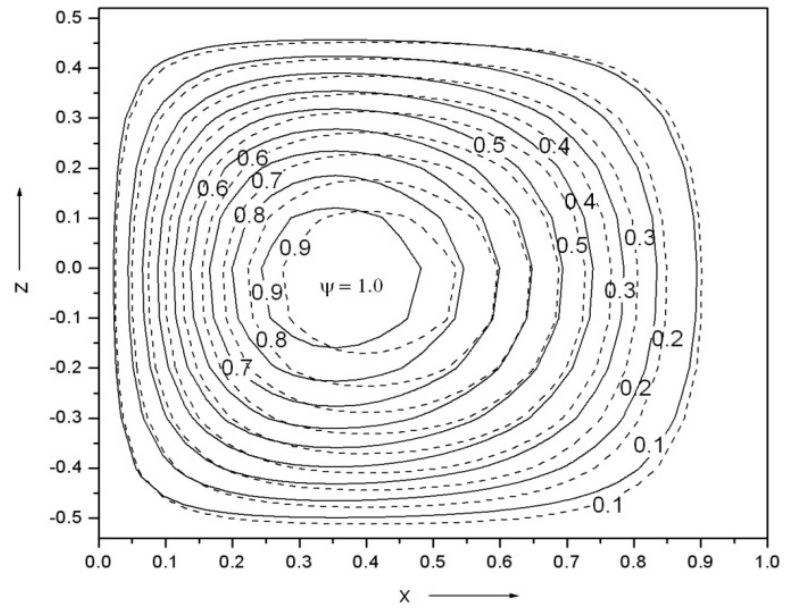

Fig.2. Comparison of the cell patterns at the onset of instability for $N=0.50$ (shown by broken curve) and $N=0.75$ (shown by continuous curve) at constant $\mu=-0.25$.

In Fig. 1 and 2, the cell patterns are shown for the cases of co-rotating cylinders and counter rotating cylinders respectively. From both figures, we found that the cells shift from outer cylinder toward the inner cylinder as $\mathrm{N}$ increases. Physically, this is true because the convection currents are flowing from the outer cylinder toward the inner one, and hence the shifting phenomenon is seen from the outer cylinder toward the inner cylinder, when $\mathrm{N}$ increases. This also confirms the destabilization of flow as 
$N$ increases. From Fig.2, we also see that as we increase the value of $\mathrm{N}$ the left hand edges of the cells becomes closer toward the inner most cell. Also, the left hand edge of the innermost cell is straightened and the corners are formed at the upper and lower ends of the innermost cell, and if $\mathrm{N}$ is further raised, the cells will start breaking through these corners, making flow unstable.

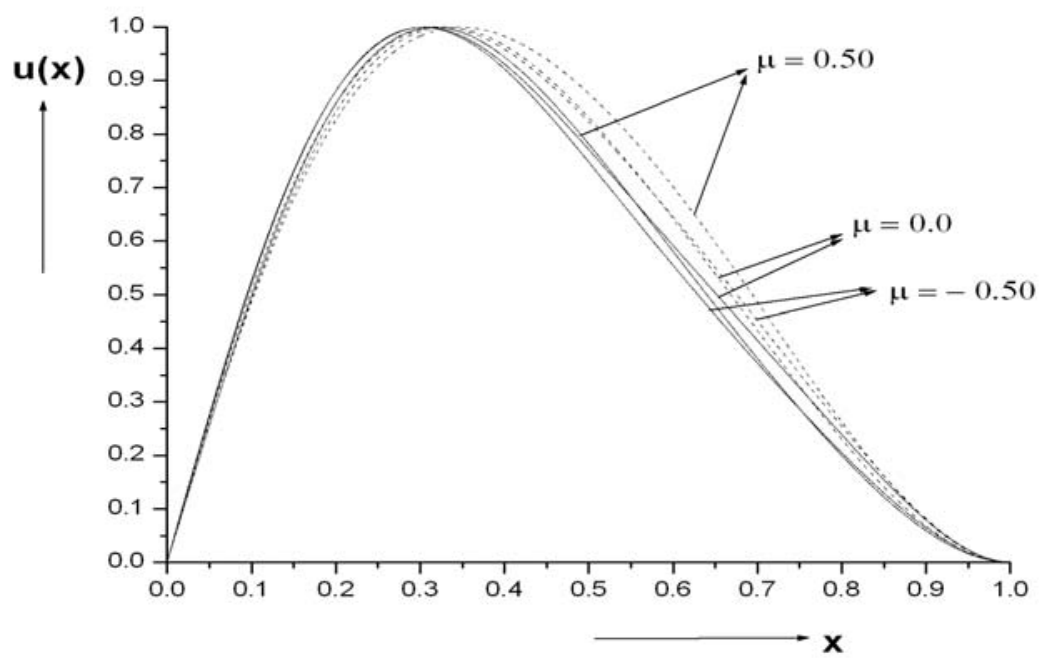

Fig.3. The radial eigen-function $\mathrm{u}(\mathrm{x})$ for $N=0.0$ (shown by continuous curve) and for $N=0.50$ (shown by broken curve) for different values of $\mu$.

In Fig. 3, the radial eigen-function $u(x)$ is shown when $N=0.0$ and $N=0.50$ for different values of $\mu$. We found that, the maximum value of $u(x)$ for $N=0.0$ is found at $x=0.3057,0.3164,0.3026$ and for $N=0.50$ is found at $x=0.3302,0.3479,0.3229$, when $\mu=0,0.50,-0.50$ respectively. From these we observe that for $N=0.0$, due to corotation of the two cylinders, the maximum of $u(x)$ shifts toward the outer cylinder as compared to the case of $\mu=0$, whereas in the presence of counter rotating cylinders the maximum of $u(x)$ shifts toward the inner cylinder, but in the presence of a positive radial temperature gradient, the maximum of $u(x)$ shifts more and more toward the outer cylinder as $N$ increases.

\section{Conclusions}

The stability of Couette flow of a viscous incompressible fluid between two concentric rotating cylinders is studied in the presence of a radial temperature gradient, when the outer cylinder is maintained at a constant heat flux. The following conclusions have been obtained from the analysis:-

1. The flow is more stable when the two cylinders are counter rotating.

2. The fluid flow gets destabilized owing to increasing $N$ for all value of $\mu$. The destabilizing is greater when the angular speed of outer cylinder $(\mu$ is $+v e)$ increases.

3. The flow gets stable, when angular speed of outer cylinder ( $\mu$ is -ve) increases when $N$ is constant.

4. When the cylinders are counter rotating, the maximum of $u(x)$ shifts toward the inner cylinder as compared to the case, when only inner cylinder is rotating, whereas in the presence of co-rotating cylinders the maximum of $u(x)$ shifts toward the outer cylinder,

5. In the presence of a positive radial temperature gradient, the maximum of $u(x)$ shifts more and more toward the outer cylinder as $N$ increases.

\section{Nomenclature}

$a$

$r$

$d$
Dimensionless wave number

Distance from the axis

Difference between two radii of the cylinders 


\begin{tabular}{|c|c|}
\hline$R_{1}, R_{2}$ & Radii of inner and outer cylinders respectively \\
\hline$u, v, w$ & Velocity components in $\mathrm{r}, \theta$ and $\mathrm{z}$ directions respectively \\
\hline$K$ & Thermal conductivity \\
\hline$q$ & Constant heat flux at the outer cylinder \\
\hline$R a$ & Rayleigh number \\
\hline$T a$ & Taylor number \\
\hline $\operatorname{Pr}$ & Prandtl number \\
\hline$N$ & Ratio of Rayleigh number and Taylor number $(R a / T a)$ \\
\hline$T$ & Temperature of fluid \\
\hline$T_{1}$ & Temperature of inner cylinder \\
\hline
\end{tabular}

\section{Greek symbols \\ $\beta \quad$ Coefficient of thermal expansion \\ $\Omega_{1}, \Omega_{2} \quad$ Angular velocity of the inner and outer cylinders respectively \\ $\mu \quad$ Ratio of angular velocities $\Omega_{2} / \Omega_{1}$ \\ $v \quad$ Kinematic viscosity}

\section{Acknowledgement}

The authors are thankful to the referees for their valuable suggestions to improve the quality of paper.

\section{References}

Aoki H. and Nohira H., 1967. Convective heat transfer in an annulus with an inner rotating cylinder. Bulletin of the Japan Society of Mechanical Engineers, Vol.10, pp. 523-532.

Ali M. A., Takhar H. S. and Soundalgekar V. M., 1998. Effect of radial temperature gradient on the stability of flow in a curved channel. Proceeding of Royal Society Londan A, Vol. 454, pp. 2279-2287.

Alibenyahia B., Lemaitre C., Nouar C. and Messaoudene N. A., 2012. Revisiting the stability of circular Couette flow of shear thinning fluids. Journal of Non-Newtonian Fluid Mechanics, Vol. 183-184, pp. 37-51.

Bjorklund S. and Kays W.M., 1959. The heat transfer between concentric rotating cylinders. Transaction of ASME, Journal of Heat Transfer. Vol. 81, pp. 176-186.

Becker K.M. and Kaye J., 1962. Measurements of diabetic flow in an annulus with an inner rotating cylinder. Transaction of ASME, Journal of Heat Transfer, Vol.84, pp. 97-105.

Chandrasekhar S., 1953. The stability of viscous flow between rotating cylinders. Mathematica, Vol. 1, pp. 5-13.

Chandrasekhar S., 1954. On characteristic value problems in high order differential equations which arise in studies of hydrodynamic and hydromagnetic stability. The American Mathematical Monthly, Vol. 61, pp. 32-54.

Chandrasekhar S., 1954. The stability of flow between rotating cylinders in the presence of radial temperature gradient. Journal of Rational Mechanics and Analysis, Vol. 3, pp. 181-207.

Chandrasekhar S., 1961. Hydrodynamic and Hydromagnetic Stability, Oxford University Press, Oxford.

DiPrima R.C., 1955. Application of the Galerkin method to the calculation of the stability of curved flows. Quartely Applied Mathematics Vol. 13, pp. 55-62.

Dutty R.L. and Reid W.H., 1964. On the stability of viscous flow between rotating cylinders, Part 1, Asymptotic Analysis. Journal of Fluid Mechanics, Vol. 20, pp. 81-94.

Deka R.K. and Paul A., 2013. Stability of narrow gap Taylor-Dean flow with radial heating. Journal of Computers and Fluids, Vol.82, pp. 87-94.

Eagles P.M. and Soundalgekar V.M., 1997. Stability of flow between two rotating cylinders in the presence of constant heat flux at the outer cylinder and radial temperature gradient-wide gap problem. Journal of Heat and Mass Transfer, Vol. 33, pp. 257-260.

Hass F.C. and Nissan A.H., 1961. Experimental heat transfer characteristics of a liquid in Couette motion and with Taylor vortices. Proceedings of the Royal Society of London, Series A, Vol. 261, pp. 215-226.

Ho C.Y., Nardacii J.L. and Nissan A.H., 1964. Heat transfer characteristics of fluids moving in a Taylor system of vortices. AICHE Journal, Vol. 10, pp. 194-202.

Harris D.L. and Reid W.H., 1964. On the stability of viscous flow between rotating cylinders, Part 2, Numerical Analysis. Journal of Fluid Mechanics, Vol. 20, pp. 95-101.

Mutabazi I., Goharzadeh A. and Dumouchel, F., 2001. The circular Couette flow with a radial temperature gradient. In 12th International Couette- Taylor workshop, September 6-8, Evanston, IL USA. 
Mahapatra T. R., Nandy S. K. and Gupta A. S., 2013. Effect of radial temperature gradient on the stability of Taylor-Dean flow between two arbitrarily spaced concentric rotating cylinders. International Journal of Heat and Mass Transfer, Vol. 57, pp. 662-670.

Prasad R., Singh A.K., Pandey S., 2012a. Stability of viscous flow in a narrow-gap annulus with radial temperature gradient and constant heat flux at inner rotating cylinder. International Journal of Energy and Technology, Vol. 4, No. 29, pp. 1-8.

Pandey S. and Prasad R. 2012. Stability of a pure pressure maintained flow in a curved channel. Proceeding of The Mathematical Society, Banaras Hindu University, Vol. 28, pp. 49-53.

Prasad R.. and Pandey S., 2012b. Stability of a pure pressure maintained flow in a curved channel. Proceeding of The Mathematical Society, Banaras Hindu University, Vol. 28, pp. 43-48.

Sharman R. D., Catton I. and Ayyaswamy P., 1973. Convective heat transfer between concentric rotating cylinders. AICHE, Symposium 131, Vol. 69, pp. 118-125.

Soundalgekar V.M., Takhar H.S. and Smith T.J., 1981. Effect of radial temperature gradient of viscous flow in an annulus with a rotating inner cylinder. Würme-Stoffübertrag Vol. 15, pp. 233-238.

Singh A.K., Yusug M. and Soundalgekar V.M., 1994. Stability of flow of a viscous incompressible fluid between concentric rotating cylinders with radial temperature gradient. Indian Journal of Pure and Applied Mathematics, Vol.25, No. 4, pp. 449456.

Soleimani M. and Sadeghy K., 2011. Instability of Bingham fluids in Taylor-Dean flow between two concentric cylinders at arbitrary gap spacings. International Journal of Non-Linear Mechanics, Vol. 46, No. 7, pp. 931-937.

Taylor G.I., 1923. Stability of a viscous liquid contained between two rotating cylinders. Philosophical Transactions of the Royal Society A, Vol. 223, pp. 289-343.

Takhar H.S., Smith T.J. and Soundalagekar V.M., 1985. On the stability of flow between two rotating concentric cylinders. Journal of Mathematical Analysis and Applications, Vol. 111, pp. 349-352.

Takhar H.S., Ali M.A. and Soundalgekar V.M., 1988a. Effect of radial temperature gradient on the stability of flow in a narrowgap annulus with constant heat flux at the inner rotating cylinder, Wärme-und Stof:

Takhar H.S., Ali M.A. and Soundalgekar V.M., 1988b. Effect of radial temperature gradient on the stability of flow in a narrowgap annulus with constant heat flux at the inner rotating cylinder. Journal of Frankling Institute, Vol. 325, pp. 609-619.

Takhar H.S., Ali M.A. and Soundalgekar V.M., 1992. Stability of flow between two rotating cylinders in the presence of constant heat flux at the outer cylinder and radial temperature gradient - narrow gap problem. Transaction of ASME Journal of Applied Mechanics, Vol. 59, pp. 464-465

Walowit J. S., Tsao and DiPrima R.C., 1964. Stability of flow between arbitrarily spaced concentric cylindrical surfaces including the effects of temperature gradient. Transactions of ASME Journal of Applied Mechanics, Vol. 31, pp. 585-593.

Yih C.S., 1961. Dual role of viscosity in the instability of revolving fluids of variable density. The Physics of Fluids. Vol. 4, pp. 886-811.

Yamamoto K., Wu X., Hyakutake T. and Yanase S., 2004. Taylor-Dean flow through a curved duct of square cross section. Fluid Dynamics Research, Vol. 35, No. 2, pp. 67-86.

\section{Biographical notes}

S. Pandey is a Research Scholar in the Department of Mathematics, Banaras Hindu University, Varanasi, India. Her research interest is Hydrodynamical Stability. She has author and coauthored in five papers in the reputed journals.

A. K. Singh is working as a Professor in the Department of Mathematics, Banaras Hindu University, Varanasi, India. He earned his PhD in Mathematics from Banaras Hindu University, Varanasi, India, in 1979. He also earned his D.Sc in Mathematics from Banaras Hindu University, Varanasi, India, in 1986. His research interest include differential equation, Fluid Dynamics and Numerical analysis, Magnetohydrodynamic flows, Flow through porous media, Non-Newtonian fluids, Heat and Mass transfer and Transport processes in cavity. He is currently the president of the Mathematical Society, Banaras Hindu University, India. He has authored and coauthored over 163 papers in the reputed international and national journals.

R. Prasad is a Research Scholar in the Department of Mathematics, Banaras Hindu University, Varanasi, India. His research interest is Magnetohydrodynamical Stability. He has author and coauthored in five papers in the reputed journals.

Received August 2013

Accepted October 2014

Final acceptance in revised form December 2014 